\title{
Cluster Synchronization in Variable-Order Fractional Community Network via Intermittent Control
}

\author{
Yi Wang and Zhaoyan $\mathrm{Wu} *$ (D) \\ School of Mathematics and Statistic, Jiangxi Normal University, Nanchang 330022, China; y.wang@jxnu.edu.cn \\ * Correspondence: zhywu@jxnu.edu.cn; Tel.: +86-791-88120367
}

check for updates

Citation: Wang, Y.; Wu, Z. Cluster Synchronization in Variable-Order Fractional Community Network via Intermittent Control. Mathematics 2021, 9, 2596. https://doi.org/ $10.3390 /$ math 9202596

Academic Editors: Ravi P. Agarwal and Christopher Goodrich

Received: 21 September 2021

Accepted: 12 October 2021

Published: 15 October 2021

Publisher's Note: MDPI stays neutral with regard to jurisdictional claims in published maps and institutional affiliations.

Copyright: (C) 2021 by the authors. Licensee MDPI, Basel, Switzerland. This article is an open access article distributed under the terms and conditions of the Creative Commons Attribution (CC BY) license (https:// creativecommons.org/licenses/by/ $4.0 /)$.

\begin{abstract}
In this paper, the cluster synchronization of a variable-order fractional community network with nonidentical dynamics is investigated. For achieving the cluster synchronization, intermittent controllers are designed, and the sufficient conditions with respect to system parameters, intermittent control instants and control gains are derived based on stability theory of fractional-order system and linear matrix inequalities (LMIs). To avoid verifying the LMIs, a corresponding simple corollary is provided. Finally, a numerical example is performed to verify the derived result.
\end{abstract}

Keywords: cluster synchronization; variable-order fractional system; community network; intermittent control

\section{Introduction}

Many large-scale physical systems consisting of a large number of interactive individuals are modeled by various dynamical networks, including integer-order and fractionalorder networks [1-35]. The individuals and interactions are denoted by nodes and (undirected/directed) edges. A community structure is a typical topology structure in dynamical networks. The individuals in the same community usually have the same local dynamics and higher density of interactions. The individuals in different communities, however, have different local dynamics and lower density of interactions. Therefore, those in the same community tend to achieve the same goal and those in different communities achieve different goals, i.e., the community network achieves cluster synchronization [1-20].

Dynamical networks, as we know, are difficult and even impossible to synchronize themselves with desired goals without external control. Many kinds of control schemes are adopted to design effective controllers to achieve cluster synchronization, such as intermittent control [1-9], impulsive control [10-13], pinning control [17-19], finite-time control $[19,20]$, and so on. Intermittent control, as a typical discontinuous control scheme, has been widely used in real systems, such as heat systems, central air-conditioning, and so on. In [1], Zhou et al. investigated the cluster synchronization of a colored community integer-order network via intermittent pinning control. In [6], Liu et al. considered the cluster synchronization of a delayed integer-order network via intermittent pinning control.

On the other hand, a fractional-order system, in virtue of its memory and genetic characteristics, has been adopted to describe many physical systems, such as the fractional-order inductor [21], cohesive fracture model [22], quantum mechanics [36], and so on. Further, the synchronization and control of a fractional-order dynamical network have been widely investigated. In [32], the synchronization of fractional-order complex-variable dynamical networks is studied using an adaptive pinning control strategy based on a close center degree. In [33], the adaptive cluster synchronization of fractional-order complex networks with internal and coupling delays as well as time-varying disturbances is investigated via the fractional-order hybrid controllers. In [34], cluster synchronization for fractional-order complex network with non-delay and delay coupling is investigated through designing both static and adaptive feedback controllers. In [35], cluster synchronization of a fractional 
network with fixed order is investigated via periodically intermittent control. In the abovementioned networks, the fractional orders are fixed, i.e., the present states always depend on the states from initial to present state, and the networks have long memory. Long memory, however, heavily increases the computational cost and reduces the performance in practical applications, for example, fast image encryption [24]. On the other hand, many real systems have only short memory, i.e., some past information is not needed and memory starts from a new state rather than the initial state. Furthermore, the fractional-orders may be variable as well [24,25,30,37-45]. In [40], the nutrient-phytoplankton-zooplankton model is extended using variable-order fractional derivatives. In [42], a triaxial creep model for salt rocks based on variable-order fractional derivatives is introduced. In [44], a three-cell population cancer model with variable-order fractional derivative with power, exponential and Mittag-Leffler memories is proposed. In [45], the constitutive relation for viscoelasticity is described by a variable-order fractional system with short memory. In [25], a new fractional variable-order creep model with short memory is introduced. In [24], new variable-order fractional chaotic systems are developed for fast image encryption. In [31], short memory fractional different equations are introduced for new memristor and neural network design. In [30], the Mittag-Leffler stability analysis of tempered fractional neural networks with short memory and variable-order is investigated. Up to now, the cluster synchronization of a variable-order fractional dynamical network is seldom studied via intermittent control.

Motivated by the above discussions, we consider the cluster synchronization of a variable-order fractional dynamical network by designing proper intermittent controllers. The main contributions are two-fold: (1) We generalize the results about intermittent control and cluster synchronization of integer-order networks to a fractional-order network. (2) We design effective aperiodically intermittent controllers and derive the synchronization conditions. In Section 2, we introduce the network model and some preliminaries. In Section 3, based on the stability theory of the fractional-order system and mathematical analysis technique, we derive the sufficient conditions for achieving cluster synchronization with respect to the system parameters, intermittent control instants and control gains. In Section 4, we perform a numerical example to illustrate the effectiveness of the derived results. In Section 5, we conclude this paper.

\section{Model and Preliminaries}

In this section, some basic definitions, lemmas and notations about the Caputo fractional-order calculus are presented, which are useful throughout this paper. In addition, the mathematical model of a variable-order fractional complex network is proposed.

Definition 1 ([46]). The fractional integral of order $\alpha$ for a function $f(t)$ is defined as

$$
{ }_{t_{0}}^{C} I_{t}^{\alpha} f(t)=\frac{1}{\Gamma(\alpha)} \int_{t_{0}}^{t}(t-\tau)^{\alpha-1} f(\tau) \mathrm{d} \tau,
$$

where $t \geq t_{0}$ and $\alpha>0$. For simplicity, denote $I_{t_{0}}^{\alpha} f(t)$ as ${ }_{t_{0}}^{C} I_{t}^{\alpha} f(t)$.

Definition 2 ([46]). The Caputo fractional derivative of function $f(t)$ is defined as

$$
{ }_{t_{0}}^{C} D_{t}^{\alpha} f(t)=\frac{1}{\Gamma(m-\alpha)} \int_{t_{0}}^{t}(t-\tau)^{m-\alpha-1} f^{(m)}(\tau) \mathrm{d} \tau,
$$

where $t>t_{0}, m-1<\alpha<m, m \in \mathbb{Z}^{+}$. Let $m=1,0<\alpha<1$, then

$$
{ }_{t_{0}}^{C} D_{t}^{\alpha} f(t)=\frac{1}{\Gamma(1-\alpha)} \int_{t_{0}}^{t}(t-\tau)^{-\alpha} f^{\prime}(\tau) \mathrm{d} \tau .
$$

For simplicity, denote $D_{t_{0}}^{\alpha} f(t)$ as ${ }_{t_{0}}^{C} D_{t}^{\alpha} f(t)$. 
Lemma 1 ([46]). If $u(t), v(t) \in C^{1}\left[t_{0},+\infty\right)$ and $0<\alpha<1$, then

$$
\begin{aligned}
& \text { (i) } D_{t_{0}}^{\alpha} I_{t_{0}}^{\alpha} u(t)=u(t), \\
& \text { (ii) } I_{t_{0}}^{\alpha} D_{t_{0}}^{\alpha} u(t)=u(t)-u\left(t_{0}\right), \\
& \text { (iii) } D_{t_{0}}^{\alpha}(u(t) \pm v(t))=D_{t_{0}}^{\alpha} u(t) \pm D_{t_{0}}^{\alpha} v(t) .
\end{aligned}
$$

Lemma 2 ([47]). Let $V(t)$ be a continuous function on $\left[t_{0},+\infty\right)$ and satisfies

$$
D_{t_{0}}^{\alpha} V(t) \leqslant \theta V(t)
$$

where $0<\alpha<1$ and $\theta$ is a constant, then

$$
V(t) \leqslant V\left(t_{0}\right) E_{\alpha}\left(\theta\left(t-t_{0}\right)^{\alpha}\right)
$$

where $E_{\alpha}(\cdot)$ is the Mittag-Leffler function.

Lemma 3 ([48]). Let $x(t) \in \mathbb{R}$ be a continuous and derivable function. Then, for any time instant $t>t_{0}$,

$$
\frac{1}{2} D_{t_{0}}^{\alpha} x^{2}(t) \leqslant x(t) D_{t_{0}}^{\alpha} x(t), \forall \alpha \in(0,1)
$$

Then, when $x(t) \in \mathbb{R}^{n}$ is continuous and derivable, it implies

$$
\frac{1}{2} D_{t_{0}}^{\alpha}\left[x^{T}(t) Q x(t)\right] \leqslant x^{T}(t) Q D_{t_{0}}^{\alpha} x(t), \forall \alpha \in(0,1),
$$

where $Q \in \mathbb{R}^{n \times n}$ is a positive definite matrix.

Consider a variable-order fractional community network consisting of $N$ nodes and $m$ communities with $2 \leq m \ll N$. The set of the nodes can be divided into $\{1, \cdots, N\}=$ $\mathcal{C}_{1} \cup \mathcal{C}_{2} \cup \cdots \cup \mathcal{C}_{m}$, where

$$
\mathcal{C}_{1}=\left\{r_{0}+1, \cdots, r_{1}\right\}, \mathcal{C}_{2}=\left\{r_{1}+1, \cdots, r_{2}\right\}, \cdots, \mathcal{C}_{m}=\left\{r_{m-1}+1, \cdots, r_{m}\right\},
$$

with $r_{0}=0, r_{m}=N$.

The node dynamics of the $p$ th community is chosen as the following variable-order fractional system with short memory

$$
D_{t_{k}}^{\alpha_{k}} x_{i}(t)=f_{p}\left(x_{i}(t)\right), t \in\left[t_{k}, t_{k+1}\right], i \in C_{p},
$$

where $0<\alpha_{k}<1, k=0,1, \cdots, x_{i}(t)=\left(x_{i 1}(t), x_{i 2}(t), \cdots, x_{i n}(t)\right)^{T} \in \mathbb{R}^{n}, f_{p}: \mathbb{R}^{n} \rightarrow \mathbb{R}^{n}$ is the nonlinear vector function, the time sequence $\left\{t_{k}\right\}$ satisfies $0=t_{0}<t_{1}<\cdots<$ $t_{k}<t_{k+1}<\cdots$ and $\lim _{k \rightarrow+\infty} t_{k}=+\infty$. Figure 1 shows an example of a variable-order fractional system with short memory.

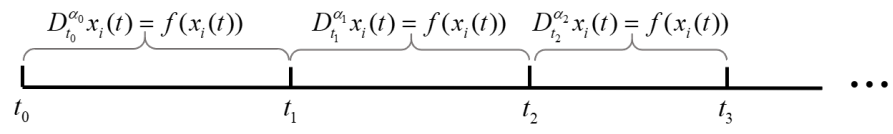

Figure 1. Variable-order fractional system with short memory.

The community network is described by

$$
D_{t_{k}}^{\alpha_{k}} x_{i}(t)=f_{p}\left(x_{i}(t)\right)+c \sum_{l=1}^{m} \sum_{j \in C_{l}} g_{i j} \Gamma x_{j}(t), t \in\left[t_{k}, t_{k+1}\right], i \in C_{p}, p=1,2, \cdots, m,
$$

$c>0$ is the coupling strengths, $\Gamma=\operatorname{diag}\left(\gamma_{1}, \gamma_{2}, \cdots, \gamma_{n}\right)>0$ is the inner coupling matrix, $G=\left(g_{i j}\right)_{N \times N}$ is the outer coupling matrix. If node $i$ is affected by node $j(j \neq i)$, 
then $g_{i j} \neq 0$; otherwise, $g_{i j}=0$, and the the diagonal elements of $G$ are defined as $g_{i i}=-\sum_{j=1, j \neq i}^{N} g_{i j}, i=1, \cdots, N$.

The matrix $G$ can be written as

$$
G=\left(\begin{array}{cccc}
G_{11} & G_{12} & \cdots & G_{1 m} \\
G_{21} & G_{22} & \cdots & G_{2 m} \\
\vdots & \vdots & \ddots & \vdots \\
G_{m 1} & G_{m 2} & \cdots & G_{m m}
\end{array}\right)
$$

where $G_{u v} \in \mathbb{R}^{\left(r_{u}-r_{u-1}\right) \times\left(r_{v}-r_{v-1}\right)}, u, v=1,2, \cdots, m$.

Definition 3 ([8]). Matrix $G=\left(g_{i j}\right) \in \mathbb{R}^{N \times N}$ is said to belong to class $\mathcal{G}_{1}$, if

$$
g_{i j} \geq 0, i \neq j, g_{i i}=-\sum_{j=1, j \neq i}^{N} g_{i j}, i=1,2, \cdots, N,
$$

and $G$ is irreducible, denoted as $G \in \mathcal{G}_{1}$.

Definition 4 ([8]). Matrix $G=\left(g_{i j}\right) \in \mathbb{R}^{N_{1} \times N_{2}}$ is said to belong to class $\mathcal{G}_{2}$ if its each row-sum is zero, i.e., $\sum_{j=1}^{N_{2}} a_{i j}=0, i=1,2, \cdots, N_{1}$, denoted as $G \in \mathcal{G}_{2}$.

Definition 5 ([8]). $G=\left(g_{i j}\right) \in \mathbb{R}^{N \times N}$, the indexes $\{1,2, \cdots, N\}$ can be divided into $m$ clusters,

$$
G=\left(\begin{array}{cccc}
G_{11} & G_{12} & \cdots & G_{1 m} \\
G_{21} & G_{22} & \cdots & G_{2 m} \\
\vdots & \vdots & \ddots & \vdots \\
G_{m 1} & G_{m 2} & \cdots & G_{m m}
\end{array}\right), G_{u v} \in \mathbb{R}^{\left(r_{u}-r_{u-1}\right) \times\left(r_{v}-r_{v-1}\right)},
$$

if $G_{u u} \in \mathcal{G}_{1}, G_{u v} \in \mathcal{G}_{2}, u, v=1,2, \cdots, m$, then the matrix is said belong to $\mathcal{G}_{3}$, denoted as $G \in \mathcal{G}_{3}$.

Let $s_{p}(t)$ be a trajectory, which is satisfied with

$$
D_{t_{k}}^{\alpha_{k}} s_{p}(t)=f_{p}\left(s_{p}(t)\right), t \in\left[t_{k}, t_{k+1}\right], p=1,2, \cdots, m .
$$

If

$$
\lim _{t \rightarrow \infty}\left\|x_{i}(t)-s_{p}(t)\right\|=0 \text { and } \lim _{t \rightarrow \infty}\left\|s_{p}(t)-s_{l}(t)\right\| \neq 0, l \neq p,
$$

where $i \in C_{p},\|\cdot\|$ is the Euclidean norm, then the network is said to achieve cluster synchronization.

For achieving the cluster synchronization of the Community Network (2), we add feedback controllers $u_{i}(t)$ to the network. The controllers work when $t \in\left[t_{2 \eta}, t_{2 \eta+1}\right]$ and rest when $t \in\left[t_{2 \eta+1}, t_{2 \eta+2}\right], \eta=0,1, \cdots$. As we know, the control scheme is called intermittent control, which is a typical discontinuous control scheme. We write the network with intermittent controllers as

$$
\begin{aligned}
D_{t_{2 \eta}}^{\alpha_{2 \eta}} x_{i}(t) & =f_{p}\left(x_{i}(t)\right)+c \sum_{l=1}^{m} \sum_{j \in C_{l}} g_{i j} \Gamma x_{j}(t)+u_{i}(t), \quad t \in\left[t_{2 \eta}, t_{2 \eta+1}\right], \\
D_{t_{2 \eta+1}}^{\alpha_{2 \eta+1}} x_{i}(t) & =f_{p}\left(x_{i}(t)\right)+c \sum_{l=1}^{m} \sum_{j \in C_{l}} g_{i j} \Gamma x_{j}(t), \quad t \in\left[t_{2 \eta+1}, t_{2 \eta+2}\right],
\end{aligned}
$$

where $i \in C_{p}, p=1,2, \cdots, m, \eta=0,1, \cdots, u_{i}(t)=-c h_{i} \Gamma\left(x_{i}(t)-s_{p}(t)\right), h_{i}>$ is feedback gain. 
Remark 1. We call $u_{i}(t)$ continuous feedback controllers if they work for $t \in\left[t_{k}, t_{k+1}\right]$ and impulsive controllers if they only work for $t=t_{k}, k=0,1,2, \cdots$. If $t_{2 \eta+2}-t_{2 \eta}$ and $t_{2 \eta+1}-t_{2 \eta}$ are constants for $\eta=0,1, \cdots$, we call $u_{i}(t)$ periodically intermittent controllers. That is, the designed controllers in this paper are aperiodically intermittent controllers.

Denote $e_{i}(t)=x_{i}(t)-s_{p}(t), i \in \mathcal{C}_{p}, E_{p}(t)=\left(e_{r_{p-1}+1}^{T}(t), \cdots, e_{r_{p}}^{T}(t)\right)^{T}, E(t)=\left(E_{1}^{T}(t)\right.$, $\left.\cdots, E_{m}^{T}(t)\right)^{T}, F_{p}\left(E_{p}\right)=\left(\left(f_{p}\left(x_{r_{p-1}+1}\right)-f_{p}\left(s_{p}\right)\right)^{T}, \cdots,\left(f_{p}\left(x_{r_{p}}\right)-f_{p}\left(s_{p}\right)\right)^{T}\right)^{T}, H_{p}=\operatorname{diag}$ $\left(h_{r_{p-1}+1}, \cdots, h_{r_{p}}\right), p=1, \cdots, m, H=\operatorname{diag}\left(H_{1}, H_{2}, \cdots, H_{m}\right)$. Then, we have the error system

$$
\begin{aligned}
D_{t_{2 \eta}}^{\alpha_{2 \eta}} E_{p}(t) & =F_{p}\left(E_{p}(t)\right)+c \sum_{l=1}^{m}\left(G_{p l} \otimes \Gamma\right) E_{l}(t)-c\left(H_{p} \otimes \Gamma\right) E_{p}(t), \quad t \in\left[t_{2 \eta}, t_{2 \eta+1}\right], \\
D_{t_{2 \eta+1}}^{\alpha_{2 \eta+1}} E_{p}(t) & =F_{p}\left(E_{p}(t)\right)+c \sum_{l=1}^{m}\left(G_{p l} \otimes \Gamma\right) E_{l}(t), \quad t \in\left[t_{2 \eta+1}, t_{2 \eta+2}\right] .
\end{aligned}
$$

Assumption 1. Suppose that there exists a positive definite matrix $\Delta=\operatorname{diag}\left(\delta_{1}, \delta_{2}, \cdots, \delta_{n}\right)$ such that

$$
(u-v)^{T}\left(f_{p}(u)-f_{p}(v)\right) \leqslant(u-v)^{T} \Delta(u-v),
$$

for any $u, v \in \mathbb{R}^{n}$.

Assumption 2. Suppose that there exist two positive constants $\delta$ and $T$ such that

$$
\left\{\begin{array}{l}
\inf _{\eta}\left(t_{2 \eta+1}-t_{2 \eta}\right)=\delta>0, \\
\sup _{\eta}\left(t_{2 \eta+2}-t_{2 \eta}\right)=T<+\infty,
\end{array}\right.
$$

where $\eta=0,1,2, \cdots$.

\section{Main Result}

In this section, we derive the sufficient conditions for achieving cluster synchronization with respect to the system parameters, intermittent control instants and control gains.

Theorem 1. Suppose Assumptions 1 and 2 hold and $G \in \mathcal{G}_{3}$. Controlled Network (4) can achieve cluster synchronization if there exist positive constants $a_{1}, a_{2}$ and $0<\epsilon<1$ such that the following inequalities hold:

$$
\begin{aligned}
& \text { (i) } I_{N} \otimes \Delta+c\left(G^{S} \otimes \Gamma\right)-c(H \otimes \Gamma)+\frac{a_{1}}{2}\left(I_{N} \otimes I_{n}\right) \leqslant 0, \\
& \text { (ii) } I_{N} \otimes \Delta+c\left(G^{S} \otimes \Gamma\right)-\frac{a_{2}}{2}\left(I_{N} \otimes I_{n}\right) \leqslant 0, \\
& \text { (iii) } E_{\alpha_{2 \eta}}\left(-a_{1} \delta^{\alpha_{2 \eta}}\right) E_{\alpha_{2 \eta+1}}\left(a_{2}(T-\delta)^{\alpha_{2 \eta+1}}\right)<\epsilon, \eta=0,1, \cdots,
\end{aligned}
$$

where $G^{S}=\frac{G+G^{T}}{2}$.

Proof. Consider the following Lyapunov function:

$$
V(t)=\frac{1}{2} \sum_{p=1}^{m} E_{p}^{T}(t) E_{p}(t) .
$$

Then the derivative of $V(t)$ along the trajectories of Equation (5) gives: 
When $t \in\left[t_{2 \eta}, t_{2 \eta+1}\right]$,

$$
\begin{aligned}
D_{t_{2 \eta}}^{\alpha_{2 \eta}} V(t) \leqslant & \sum_{p=1}^{m} E_{p}^{T}(t) D_{t_{2 \eta}}^{\alpha_{2 \eta}} E_{p}(t) \\
= & \sum_{p=1}^{m} E_{p}^{T}(t)\left(F_{p}\left(E_{p}(t)\right)+c \sum_{l=1}^{m}\left(G_{p l} \otimes \Gamma\right) E_{l}(t)-c\left(H_{p} \otimes \Gamma\right) E_{p}(t)\right) \\
\leqslant & \sum_{p=1}^{m} E_{p}^{T}(t) F_{p}\left(E_{p}(t)\right)+c \sum_{p=1}^{m} \sum_{l=1}^{m} E_{p}^{T}(t)\left(G_{p l} \otimes \Gamma\right) E_{l}(t) \\
& -c \sum_{p=1}^{m} E_{p}^{T}(t)\left(H_{p} \otimes \Gamma\right) E_{p}(t) \\
\leqslant & E^{T}(t)\left(I_{N} \otimes \Delta\right) E(t)+c E^{T}(t)\left(G^{S} \otimes \Gamma\right) E(t)-c E^{T}(t)(H \otimes \Gamma) E(t) \\
\leqslant & E^{T}(t)\left(I_{N} \otimes \Delta+c\left(G^{S} \otimes \Gamma\right)-c(H \otimes \Gamma)+\frac{a_{1}}{2}\left(I_{N} \otimes I_{n}\right)\right) E(t) \\
& -\frac{a_{1}}{2} E^{T}(t) E(t) \\
\leqslant & -a_{1} V(t) .
\end{aligned}
$$

By Lemma 2, we have

$$
V(t) \leqslant V\left(t_{2 \eta}\right) E_{\alpha_{2 \eta}}\left(-a_{1}\left(t-t_{2 \eta}\right)^{\alpha_{2 \eta}}\right), t \in\left[t_{2 \eta}, t_{2 \eta+1}\right]
$$

and

$$
V\left(t_{2 \eta+1}\right) \leqslant V\left(t_{2 \eta}\right) E_{\alpha_{2 \eta}}\left(-a_{1}\left(t_{2 \eta+1}-t_{2 \eta}\right)^{\alpha_{2 \eta}}\right) .
$$

Similarly, when $t \in\left[t_{2 \eta+1}, t_{2 \eta+2}\right]$,

$$
\begin{aligned}
D_{t_{2 \eta+1}}^{\alpha_{2 \eta+1}} V(t) & \leqslant \sum_{p=1}^{m} E_{p}^{T}(t) D_{t_{2 \eta+1}}^{\alpha_{p}} E_{p}(t) \\
& =\sum_{p=1}^{m} E_{p}^{T}(t)\left(F_{p}\left(E_{p}(t)\right)+c \sum_{l=1}^{m}\left(G_{p l} \otimes \Gamma\right) E_{l}(t) E_{p}(t)\right) \\
& \leqslant \sum_{p=1}^{m} E_{p}^{T}(t) F_{p}\left(E_{p}(t)\right)+c \sum_{p=1}^{m} \sum_{l=1}^{m} E_{p}^{T}(t)\left(G_{p l} \otimes \Gamma\right) E_{l}(t) \\
& \leqslant E^{T}(t)\left(I_{N} \otimes \Delta\right) E(t)+c E^{T}(t)\left(G^{S} \otimes \Gamma\right) E(t) \\
& \leqslant E^{T}(t)\left(I_{N} \otimes \Delta+c\left(G^{S} \otimes \Gamma\right)-\frac{a_{2}}{2}\left(I_{N} \otimes I_{n}\right)\right) E(t)+\frac{a_{2}}{2} E^{T}(t) E(t) \\
& \leqslant a_{2} V(t)
\end{aligned}
$$

which implies,

$$
V(t) \leqslant V\left(t_{2 \eta+1}\right) E_{\alpha_{2 \eta+1}}\left(a_{2}\left(t-t_{2 \eta+1}\right)^{\alpha_{2 \eta+1}}\right)
$$

and

$$
\begin{aligned}
V\left(t_{2 \eta+2}\right) & \leqslant V\left(t_{2 \eta+1}\right) E_{\alpha_{2 \eta+1}}\left(a_{2}\left(t_{2 \eta+2}-t_{2 \eta+1}\right)^{\alpha_{2 \eta+1}}\right) \\
& \leqslant V\left(t_{2 \eta}\right) E_{\alpha_{2 \eta}}\left(-a_{1}\left(t_{2 \eta+1}-t_{2 \eta}\right)^{\alpha_{2 \eta}}\right) E_{\alpha_{2 \eta+1}}\left(a_{2}\left(t_{2 \eta+2}-t_{2 \eta+1}\right)^{\alpha_{2 \eta+1}}\right) .
\end{aligned}
$$


Thus, for any positive integer $l$, we have

$$
\begin{aligned}
V\left(t_{2 l}\right) & \leqslant V(0) \prod_{\eta=0}^{l-1} E_{\alpha_{2 \eta}}\left(-a_{1}\left(t_{2 \eta+1}-t_{2 \eta}\right)^{\alpha_{2 \eta}}\right) E_{\alpha_{2 \eta+1}}\left(a_{2}\left(t_{2 \eta+2}-t_{2 \eta+1}\right)^{\alpha_{2 \eta+1}}\right) \\
& \leqslant V(0) \prod_{\eta=0}^{l-1} E_{\alpha_{2 \eta}}\left(-a_{1} \delta^{\alpha_{2 \eta}}\right) E_{\alpha_{2 \eta+1}}\left(a_{2}(T-\delta)^{\alpha_{2 \eta+1}}\right) \\
& \leqslant V(0) \epsilon^{l}
\end{aligned}
$$

and $\lim _{l \rightarrow+\infty} V\left(t_{2 l}\right)=0$. Then, for $t \in\left[t_{2 l}, t_{2 l+1}\right]$, we have $V(t) \leqslant V\left(t_{2 l}\right) E_{\alpha_{2 l}}\left(-a_{1}(t-\right.$ $\left.t_{2 l}\right)^{\alpha_{2 l}}$ ) from Equation (6), i.e., $\lim _{l \rightarrow+\infty} V(t)=0$ and $\lim _{l \rightarrow+\infty} V\left(t_{2 l+1}\right)=0$. Similarly, for $t \in\left[t_{2 l+1}, t_{2 l+2}\right]$, we also have $\lim _{l \rightarrow+\infty} V(t)=0$ from Equation (7). Therefore, $\lim _{t \rightarrow+\infty} V(t)=0$, i.e., $\lim _{t \rightarrow+\infty}\left\|e_{i}(t)\right\|=0$ and the cluster synchronization is achieved.

Let $\delta^{*}=\max \left\{\delta_{i}, i=1,2, \cdots, n\right\}, g^{*}=\lambda_{\max }\left(G^{S} \otimes \Gamma\right)$, and $h^{*}=\lambda_{\max }(H \otimes \Gamma)$, we have the following corollary.

Corollary 1. Suppose all Assumptions in Theorem 1 hold. Controlled Network (4) can achieve cluster synchronization if there exist positive constants $a_{1}, a_{2}$ and $0<\epsilon<1$ such that the following inequalities hold:

$$
\begin{aligned}
& \text { (i) } \delta^{*}+c g^{*}-c h^{*}+\frac{a_{1}}{2} \leqslant 0, \\
& \text { (ii) } \delta^{*}+c g^{*}-\frac{a_{2}}{2} \leqslant 0, \\
& \text { (iii) } E_{\alpha_{2 \eta}}\left(-a_{1} \delta^{\alpha_{2 \eta}}\right) E_{\alpha_{2 \eta+1}}\left(a_{2}(T-\delta)^{\alpha_{2 \eta+1}}\right)<\epsilon, \eta=0,1, \cdots .
\end{aligned}
$$

Remark 2. For any given Network (4), by simple calculations, we can choose proper $a_{2}$ satisfying condition (ii) in Theorem 1, and then choose a proper $a_{1}$ satisfying condition (iii). Finally, we can choose feedback gains $h_{i}$ such that condition (i) holds.

Remark 3. In this paper, the variable-order function is a piecewise constant function. When $\alpha_{k}=\alpha$ for all $k=0,1, \cdots$, the variable-order fractional network degenerates to a conventional fractional-order network in [49]. Furthermore, condition (iii) in Theorem 1 (or Corollary 1) is rewritten as $E_{\alpha}\left(-a_{1} \delta^{\alpha}\right) E_{\alpha}\left(a_{2}(T-\delta)^{\alpha}\right)<\epsilon$, which is similar to condition (iii) in Theorem 1 of Ref. [49]. That is, the obtained results generalize the results in Ref. [49] from constant order to variable order.

Remark 4. If we choose $\alpha_{k}=1, k=0,1, \cdots$, then Network (4) is an integer-order network, and condition (iii) in Theorem 1 (or Corollary 1) is rewritten as $e^{-a_{1} \delta+a_{2}(T-\delta)}<\epsilon<1$. Further, we have $-a_{1} \delta+a_{2}(T-\delta)=\ln \epsilon<0$, which is similar to the condition in Theorem 1 of Ref. [8]. That is, we generalize the results in Ref. [8] from integer-order network to fractional-order network.

\section{Simulation Results}

Consider a variable-order fractional community network consisting of 19 nodes and 3 communities. The topology of the network is shown in Figure 2.

The node dynamics of the $p$ th community is chosen as a variable-order Chen system [24]:

$$
\left\{\begin{array}{l}
D_{t_{k}}^{\alpha_{k}} x_{i 1}=\widetilde{a}_{p}\left(x_{i 2}-x_{i 1}\right), \\
D_{t_{k}}^{\alpha_{k}} x_{i 2}=\left(\widetilde{c}-\widetilde{a}_{p}\right) x_{i 1}-x_{i 1} x_{i 3}+\widetilde{c} x_{i 2}, \\
D_{t_{k}}^{\alpha_{k}} x_{i 3}=x_{i 1} x_{i 2}-\widetilde{b} x_{i 3},
\end{array}\right.
$$


where $i=1,2, \cdots, 19, p=1,2,3, k=0,1, \cdots, 0<\alpha_{k}<1$. The coefficients are $\widetilde{a}_{1}=30$, $\widetilde{a}_{2}=35, \widetilde{a}_{3}=40, \widetilde{b}=3$ and $\widetilde{c}=28$. According to [50,51], when $0.93 \leqslant \alpha_{k} \leqslant 0.99$, the variable-order Chen system exhibits a chaotic orbit.

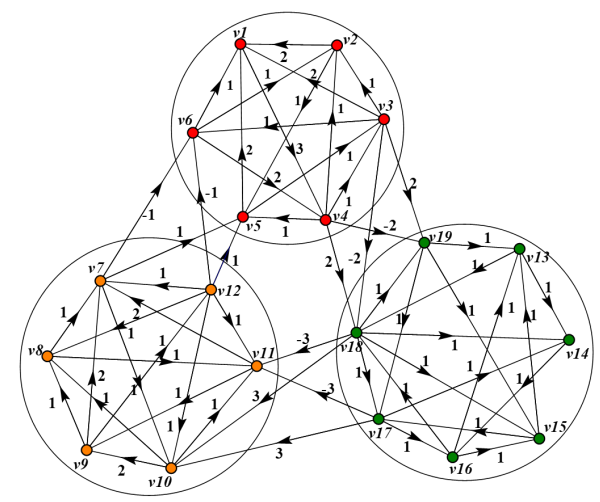

Figure 2. The outer coupling matrix $G$.

The variable orders $\alpha_{k}$ of the variable-order fractional Chen system (System (8)) are chosen, as shown in Table 1, and the chaotic orbits are shown in Figure 3. From Figure 3, we have $\left(\left|s_{11}\right|,\left|s_{12}\right|,\left|s_{13}\right|\right)<(24,28,54),\left(\left|s_{21}\right|,\left|s_{22}\right|,\left|s_{23}\right|\right)<(21,24,38),\left(\left|s_{31}\right|,\left|s_{32}\right|,\left|s_{33}\right|\right)<$ $(17,18,28)$.

Table 1. The variable orders in System (8).

\begin{tabular}{ccccccccc}
\hline$k$ & 0 & 1 & 2 & 3 & 4 & 5 & 6 & 7 \\
\hline$\alpha_{k}$ & 0.99 & 0.97 & 0.98 & 0.96 & 0.99 & 0.93 & 0.95 & 0.94 \\
\hline$k$ & 8 & 9 & 10 & 11 & 12 & 13 & 14 & 15 \\
\hline$\alpha_{k}$ & 0.97 & 0.95 & 0.96 & 0.95 & 0.97 & 0.99 & 0.98 & 0.98 \\
\hline
\end{tabular}

For the node dynamics of the $p$ th community, we have

$$
\begin{aligned}
& \left(x_{i}-s_{p}\right)^{T}\left(f_{p}\left(x_{i}\right)-f_{p}\left(s_{p}\right)\right) \\
& =e_{i}^{T}\left(\widetilde{a}_{p} e_{i 2}-\widetilde{a} e_{i 1}(\widetilde{c}-\widetilde{a}) e_{i 1}-x_{i 1} x_{i 3}+s_{p 1} s_{p 3}+\widetilde{c} e_{i 2}, x_{i 1} x_{i 2}-s_{p 1} s_{p 2}-\widetilde{b} e_{i 3}\right)^{T} \\
& =\widetilde{a}_{p} e_{i 1} e_{i 2}-\widetilde{a} e_{i 1}^{2}+(\widetilde{c}-\widetilde{a}) e_{i 1} e_{i 2}+e_{i 2}\left(-x_{i 1} e_{i 3}-e_{i 1} s_{p 3}\right)+\widetilde{c} e_{i 2}^{2}+e_{i 3}\left(x_{i 1} e_{i 2}+e_{i 1} s_{p 2}\right)-\widetilde{b} e_{i 3}^{2} \\
& =-\widetilde{a}_{p} e_{i 1}^{2}+\widetilde{c} e_{i 2}^{2}-\widetilde{b} e_{i 3}^{2}+\widetilde{c} e_{i 1} e_{i 2}-s_{p 3} e_{i 1} e_{i 2}+s_{p 2} e_{i 1} e_{i 3} \\
& \leqslant\left(-\widetilde{a}_{p}+\frac{\widetilde{c}+\left|s_{p 3}\right|}{2 \eta}+\frac{\left|s_{p 2}\right|}{2 \rho}\right) e_{i 1}^{2}+\left(\widetilde{c}+\frac{\widetilde{c}+\left|s_{p 3}\right|}{2} \eta\right) e_{i 2}^{2}+\left(-\widetilde{b}+\frac{\left|s_{p 2}\right|}{2} \rho\right) e_{i 3}^{2} \\
& \leqslant \lambda_{p}\left(x_{i}-s_{p}\right)^{T}\left(x_{i}-s_{p}\right),
\end{aligned}
$$

where $\lambda_{p}=\max \left\{-\widetilde{a}_{p}+\frac{\widetilde{c}+\left|s_{p 3}\right|}{2 \eta}+\frac{\left|s_{p 2}\right|}{2 \rho}, \widetilde{c}+\frac{\widetilde{c}+\left|s_{p 3}\right|}{2} \eta,-\widetilde{b}+\frac{\left|s_{p 2}\right|}{2} \rho\right\}$. By simple calculations, we can choose $\Delta=\operatorname{diag}(50.14,50.14,50.14)$ such that Assumption 1 holds. 

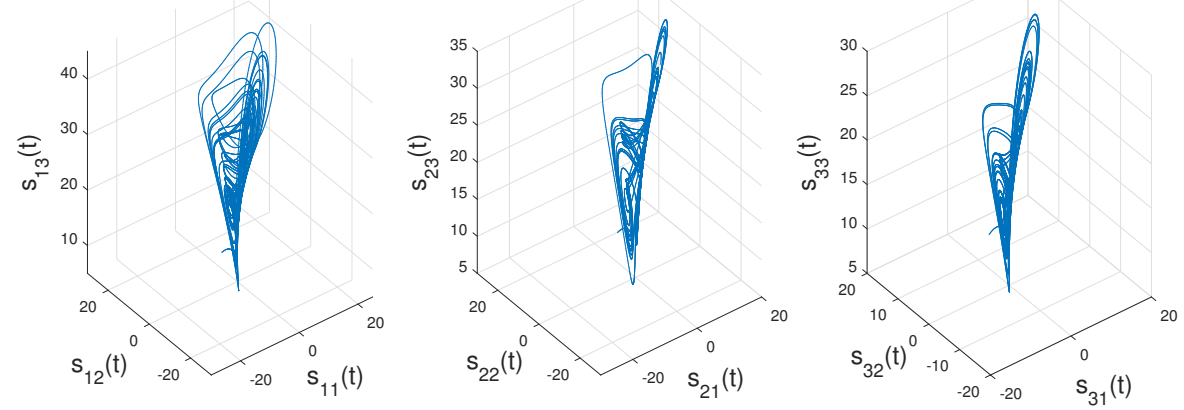

Figure 3. The chaotic behavior of variable-order fractional Chen system (System (8)) with $x_{i}(0)=$ $(-9,-5,14)^{T}$.

The network with intermittent controllers is written as

$$
\left\{\begin{array}{l}
D_{t_{2 \eta}}^{\alpha_{2 \eta}} x_{i}(t)=f_{p}\left(x_{i}(t)\right)+c \sum_{l=1}^{m} \sum_{j \in C_{l}} g_{i j} \Gamma x_{j}(t)-c h_{i} \Gamma\left(x_{i}(t)-s_{p}(t)\right), \quad t \in\left[t_{2 \eta}, t_{2 \eta+1}\right], \\
D_{t_{2 \eta+1}}^{\alpha_{2 \eta+1}} x_{i}(t)=f_{p}\left(x_{i}(t)\right)+c \sum_{l=1}^{m} \sum_{j \in C_{l}} g_{i j} \Gamma x_{j}(t), \quad t \in\left[t_{2 \eta+1}, t_{2 \eta+2}\right] .
\end{array}\right.
$$

We choose $\delta=\inf _{\eta}\left(t_{2 \eta+1}-t_{2 \eta}\right)=0.04>0, T=\sup _{\eta}\left(t_{2 \eta+2}-t_{2 \eta}\right)=0.06<+\infty$ in Assumption 2.

In numerical simulations, we choose $c=1, \Gamma$ as an identity matrix, the time sequence $\left\{t_{k}\right\}$ in Table 2 , the initial values are $s_{p}(0)=(-9,-5,14)^{T}, p=1,2,3 x_{i}(0)=(25 \sin (3(i-$ $1)+1), 25 \sin (3(i-1)+2), 25 \sin (3(i-1)+3))^{T}$. By simple calculations, we have $g^{*}=$ 0.9636. Then we choose $h^{*}=89, a_{1}=74.5$ and $a_{2}=103$ such that

$$
\begin{aligned}
& \text { (i) } \delta^{*}+c g^{*}-c h^{*}+\frac{a_{1}}{2}=-0.6464 \leqslant 0, \\
& \text { (ii) } \delta^{*}+c g^{*}-\frac{a_{2}}{2}=-0.3964 \leqslant 0, \\
& \text { (iii) } E_{\alpha_{2 \eta}}\left(-a_{1} \delta^{\alpha_{2 \eta}}\right) E_{\alpha_{2 \eta+1}}\left(a_{2}(T-\delta)^{\alpha_{2 \eta+1}}\right) \leqslant 0.9910<1 .
\end{aligned}
$$

i.e., the conditions in Corollary 1 are satisfied, and cluster synchronization is achieved. The trajectories of $x_{i j}$ and $s_{p j}, i=1,2, \cdots, 19, j=1,2,3, p=1,2,3$ are shown in Figure 4 . 

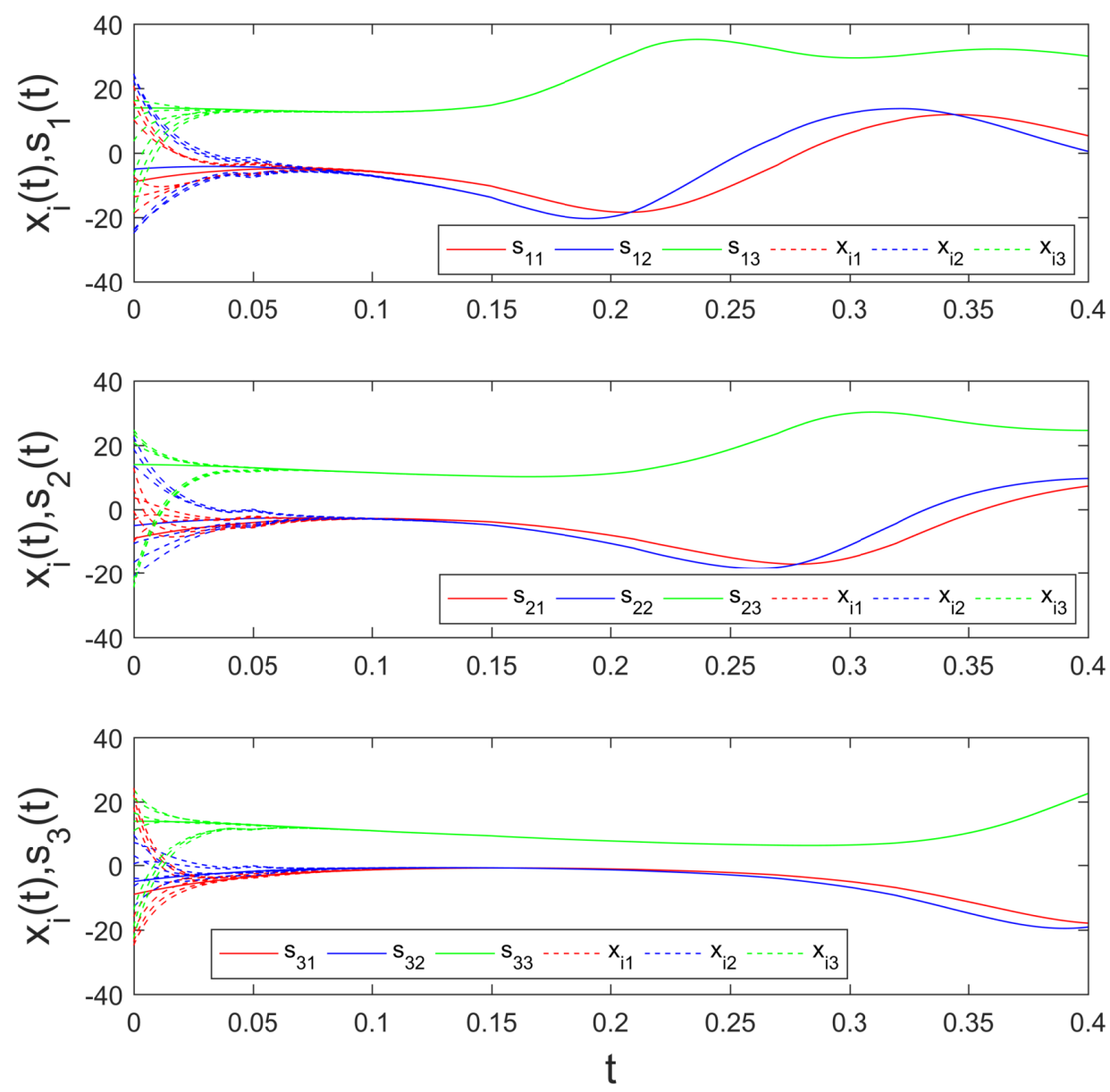

Figure 4. The top shows the trajectories of $x_{i j}$ and $s_{1 j}, i=1,2, \cdots, 6, j=1,2,3$. The middle shows the trajectories of $x_{i j}$ and $s_{2 j}, i=7,8, \cdots, 12, j=1,2,3$. The bottem shows the trajectories of $x_{i j}$ and $s_{3 j}, i=13,14, \cdots, 19, j=1,2,3$.

Table 2. The time sequence $\left\{t_{k}\right\}$.

\begin{tabular}{ccccccccc}
\hline$k$ & 0 & 1 & 2 & 3 & 4 & 5 & 6 & 7 \\
\hline$t_{k}$ & 0 & 0.04 & 0.05 & 0.10 & 0.11 & 0.15 & 0.17 & 0.21 \\
\hline$k$ & 8 & 9 & 10 & 11 & 12 & 13 & 14 & 15 \\
\hline$t_{k}$ & 0.22 & 0.27 & 0.28 & 0.32 & 0.34 & 0.38 & 0.40 & 0.42 \\
\hline
\end{tabular}

\section{Conclusions}

The cluster synchronization of a variable-order fractional community network is studied via intermittent control. The local dynamics of different communities are nonidentical. The synchronization conditions are derived according to the stability theory of a fractionalorder system and LMIs. Furthermore, the obtained result is verified to be correct by a numerical example.

Author Contributions: Y.W. wrote the original draft preparation. Z.W. reviewed and edited the whole paper. All authors have read and agreed to the published version of the manuscript.

Funding: This work was jointly supported by the NSFC Grant Nos. 61963019 and 61463022, the NSF for Distinguished Young Scholar of Jiangxi Province of China Grant No. 20171BCB23031, and the Graduate Innovation Fund of Jiangxi Normal University. 
Institutional Review Board Statement: Not applicable.

Informed Consent Statement: Not applicable.

Data Availability Statement: The data can be found in the manuscript.

Conflicts of Interest: The authors declare no conflict of interest.

\section{References}

1. Zhou, P.; Cai, S.; Shen, J.; Liu, Z. Adaptive exponential cluster synchronization in colored community networks via aperiodically intermittent pinning control. Nonlinear Dyn. 2018, 92, 905-921. [CrossRef]

2. Yang, Q.; Wu, H.; Cao, J. Global cluster synchronization in finite time for complex dynamical networks with hybrid couplings via aperiodically intermittent control. Optim. Control. Appl. Methods 2020, 41, 1097-1117. [CrossRef]

3. Feng, J.; Yang, P.; Zhao, Y. Cluster synchronization for nonlinearly time-varying delayed coupling complex networks with stochastic perturbation via periodically intermittent pinning control. Appl. Math. Comput. 2016, 291, 52-68. [CrossRef]

4. Cai, S.; Jia, Q.; Liu, Z. Cluster synchronization for directed heterogeneous dynamical networks via decentralized adaptive intermittent pinning control. Nonlinear Dyn. 2015, 82, 689-702. [CrossRef]

5. Guan, Z.H.; Yue, D.; Hu, B.; Li, T.; Liu, F. Cluster Synchronization of Coupled Genetic Regulatory Networks with Delays via Aperiodically Adaptive Intermittent Control. IEEE Trans. Nanobiosci. 2017, 16, 585-599. [CrossRef]

6. Liu, X.; Li, P.; Chen, T. Cluster synchronization for delayed complex networks via periodically intermittent pinning control. Neurocomputing 2015, 162, 191-200. [CrossRef]

7. Chen, H.; Shi, P.; Lim, C.C. Cluster Synchronization for Neutral Stochastic Delay Networks via Intermittent Adaptive Control. IEEE Trans. Neural Netw. Learn. Syst. 2019, 30, 3246-3259. [CrossRef]

8. Liu, X.; Chen, T. Cluster synchronization in directed networks via intermittent pinning control. IEEE Trans. Neural Netw. 2011, 22, 1009-1020. [CrossRef]

9. Kang, Y.; Qin, J.; Ma, Q.; Gao, H.; Zheng, W.X. Cluster synchronization for interacting clusters of nonidentical nodes via intermittent pinning control. IEEE Trans. Neural Netw. Learn. Syst. 2018, 29, 1747-1759. [CrossRef] [PubMed]

10. Liu, L.; Liu, K.; Xiang, H.; Liu, Q. Pinning impulsive cluster synchronization of complex dynamical network. Phys. A Stat. Mech. Its Appl. 2020, 545, 123580. [CrossRef]

11. Pan, L.; Cao, J.; Al-Juboori, U.A.; Abdel-Aty, M. Cluster synchronization of stochastic neural networks with delay via pinning impulsive control. Neurocomputing 2019, 366, 109-117. [CrossRef]

12. Gong, X.; Gan, L.; Wu, Z. Adaptive impulsive cluster synchronization in community network with nonidentical nodes. Int. J. Mod. Phys. C 2016, 27, 1650010. [CrossRef]

13. Wang, X.; Park, J.H.; Yang, H.; Zhong, S. An improved impulsive control approach for cluster synchronization of complex networks with parameter mismatches. IEEE Trans. Syst. Man Cybern. Syst. 2021, 51, 2561-2570. [CrossRef]

14. Du, K.; Ma, Q.; Kang, Y.; Fu, W. Robust Cluster Synchronization in Dynamical Networks With Directed Switching Topology via Averaging Method. IEEE Trans. Syst. Man Cybern. Syst. 2020. [CrossRef]

15. Zhou, L.; Tan, F.; Yu, F.; Liu, W. Cluster synchronization of two-layer nonlinearly coupled multiplex networks with multi-links and time-delays. Neurocomputing 2019, 359, 264-275. [CrossRef]

16. Menara, T.; Baggio, G.; Bassett, D.S.; Pasqualetti, F. Stability Conditions for Cluster Synchronization in Networks of Heterogeneous Kuramoto Oscillators. IEEE Trans. Control Netw. Syst. 2020, 7, 302-314. [CrossRef]

17. Liu, L.; Ding, X.; Zhou, W. Prescribed-time cluster synchronization of uncertain complex dynamical networks with switching via pinning control. Neurocomputing 2021, 419, 136-147. [CrossRef]

18. Zhang, J.; Ma, Z.; Li, X.; Qiu, J. Cluster Synchronization in Delayed Networks With Adaptive Coupling Strength via Pinning Control. Front. Phys. 2020, 8, 235. [CrossRef]

19. Liu, X.; Chen, T. Finite-time and fixed-time cluster synchronization with or without pinning control. IEEE Trans. Cybern. 2018, 48, 240-252. [CrossRef]

20. Liu, P.; Zeng, Z.; Wang, J. Asymptotic and Finite-Time Cluster Synchronization of Coupled Fractional-Order Neural Networks with Time Delay. IEEE Trans. Neural Netw. Learn. Syst. 2020, 31, 4956-4967. [CrossRef]

21. Zhang, L.; Kartci, A.; Elwakil, A.; Bagci, H.; Salama, K.N. Fractional-Order Inductor: Design, Simulation, and Implementation. IEEE Access 2021, 9, 73695-73702. [CrossRef]

22. Giraldo-Londoño, O.; Paulino, G.H.; Buttlar, W.G. Fractional calculus derivation of a rate-dependent PPR-based cohesive fracture model: Theory, implementation, and numerical results. Int. J. Fract. 2019, 216, 1-29. [CrossRef]

23. Wu, G.C.; Zeng, D.Q.; Baleanu, D. Fractional impulsive differential equations: Exact solutions, integral equations and short memory case. Fract. Calc. Appl. Anal. 2019, 22, 180-192. [CrossRef]

24. Wu, G.C.; Deng, Z.G.; Baleanu, D.; Zeng, D.Q. New variable-order fractional chaotic systems for fast image encryption. Chaos 2019, 29, 083103. [CrossRef]

25. Wu, F.; Gao, R.; Liu, J.; Li, C. New fractional variable-order creep model with short memory. Appl. Math. Comput. 2020, 380, 125278. [CrossRef]

26. Xu, L.; Chu, X.; Hu, H. Quasi-synchronization analysis for fractional-order delayed complex dynamical networks. Math. Comput. Simul. 2021, 185, 594-613. [CrossRef] 
27. Zhou, J.; Yan, J.; Wu, Z. Synchronization of fractional-order dynamical network via aperiodically intermittent pinning control. Adv. Differ. Equ. 2019, 2019, 165. [CrossRef]

28. Liu, P.; Zeng, Z.; Wang, J. Global Synchronization of Coupled Fractional-Order Recurrent Neural Networks. IEEE Trans. Neural Netw. Learn. Syst. 2019, 30, 2358-2368. [CrossRef]

29. Zhang, F.; Zeng, Z. Multistability of Fractional-Order Neural Networks with Unbounded Time-Varying Delays. IEEE Trans. Neural Netw. Learn. Syst. 2021, 32, 177-187. [CrossRef] [PubMed]

30. Gu, C.Y.; Zheng, F.X.; Shiri, B. Mittag-leffler stability analysis of tempered fractional neural networks with short memory and variable-order. Fractals 2021, 2140029. [CrossRef]

31. Wu, G.C.; Luo, M.; Huang, L.L.; Banerjee, S. Short memory fractional differential equations for new memristor and neural network design. Nonlinear Dyn. 2020, 100, 3611-3623. [CrossRef]

32. Ding, D.W.; Yan, J.; Wang, N.; Liang, D. Adaptive Synchronization of Fractional Order Complex-Variable Dynamical Networks via Pinning Control. Commun. Theor. Phys. 2017, 68, 366-374. [CrossRef]

33. Fan, H.; Zhao, Y. Cluster Synchronization of Fractional-Order Nonlinearly-Coupling Community Networks with Time-Varying Disturbances and Multiple Delays. IEEE Access 2021, 9, 60934-60945. [CrossRef]

34. Wang, Y.; Wu, Z. Cluster synchronization in fractional-order network with nondelay and delay coupling. Int. J. Mod. Phys. C 2021, 2250006. [CrossRef]

35. Zhou, J.; Zhao, Y.; Wu, Z.Y. Cluster synchronization of fractional-order directed networks via intermittent pinning control. Phys. A Stat. Mech. Its Appl. 2019, 519, 22-33. [CrossRef]

36. Zhang, X.; Wei, C.; Liu, Y.; Luo, M. Fractional corresponding operator in quantum mechanics and applications: A uniform fractional Schrödinger equation in form and fractional quantization methods. Ann. Phys. 2014, 350, 124-136. [CrossRef]

37. Zhou, F.X.; Wang, L.Y.; Liu, Z.Y.; Zhao, W.C. A viscoelastic-viscoplastic mechanical model of time-dependent materials based on variable-order fractional derivative. Mech. Time-Depend. Mater. 2021. [CrossRef]

38. Liu, J.; Wu, F.; Zou, Q.; Chen, J.; Ren, S.; Zhang, C. A variable-order fractional derivative creep constitutive model of salt rock based on the damage effect. Geomech. Geophys. Geo-Energy Geo-Resour. 2021, 7. [CrossRef]

39. Dehestani, H.; Ordokhani, Y.; Razzaghi, M. Application of fractional Gegenbauer functions in variable-order fractional delay-type equations with non-singular kernel derivatives. Chaos Solitons Fractals 2020, 140, 110111. [CrossRef]

40. Ghanbari, B.; Gómez-Aguilar, J.F. Modeling the dynamics of nutrient-phytoplankton-zooplankton system with variable-order fractional derivatives. Chaos Solitons Fractals 2018, 116, 114-120. [CrossRef]

41. Tang, H.; Wang, D.; Huang, R.; Pei, X.; Chen, W. A new rock creep model based on variable-order fractional derivatives and continuum damage mechanics. Bull. Eng. Geol. Environ. 2018, 77, 375-383. [CrossRef]

42. Fei, W.; Jie, L.; Quanle, Z.; Cunbao, L.; Jie, C.; Renbo, G. A triaxial creep model for salt rocks based on variable-order fractional derivative. Mech. Time-Depend. Mater. 2021, 25, 101-118. [CrossRef]

43. Xiang, M.; Yang, D.; Zhang, B. Homoclinic solutions for Hamiltonian systems with variable-order fractional derivatives. Complex Var. Elliptic Equ. 2020, 65, 1412-1432. [CrossRef]

44. Kachia, K.; Solís-Pérez, J.E.; Gómez-Aguilar, J.F. Chaos in a three-cell population cancer model with variable-order fractional derivative with power, exponential and Mittag-Leffler memories. Chaos Solitons Fractals 2020, 140, 110177. [CrossRef]

45. Ramirez, L.E.; Coimbra, C.F. A variable order constitutive relation for viscoelasticity. Ann. Der Phys. (Leipz.) 2007, 16, 543-552. [CrossRef]

46. Kilbas, A.; Srivastava, H.; Trujillo, J. Theory and Application of Fractional Differential Equations; Elsevier: Amsterdam, The Netherlands, 2006. [CrossRef]

47. Podlubny, I. Fractional Differential Equations: An Introduction to Fractional Derivatives, Fractional Differential Equations, to Methods of Their Solution (Mathematics in Science and Engineering); Elsevier: Amsterdam, The Netherlands, 1999.

48. Aguila-Camacho, N.; Duarte-Mermoud, M.A.; Gallegos, J.A. Lyapunov functions for fractional order systems. Commun. Nonlinear Sci. Numer. Simul. 2014, 19, 2951-2957. [CrossRef]

49. Wang, F.; Yang, Y. Intermittent synchronization of fractional order coupled nonlinear systems based on a new differential inequality. Phys. A Stat. Mech. Its Appl. 2018, 512, 142-152. [CrossRef]

50. Lu, J.G.; Chen, G. A note on the fractional-order Chen system. Chaos Solitons Fractals 2006, 27, 685-688. [CrossRef]

51. Li, C.; Chen, G. Chaos in the fractional order Chen system and its control. Chaos Solitons Fractals 2004, 22, 549-554. [CrossRef] 